\title{
Understanding the cost savings of video visits in outpatient surgical clinics
}

\author{
David S. Portney ${ }^{1,2} \wedge$, Rohan Ved ${ }^{3}$, Vahagn Nikolian ${ }^{4} \wedge$, Andrea $\mathrm{Wei}^{4}$, Tom Buchmueller ${ }^{5}$, Brad Killaly ${ }^{5}$, \\ Hasan B. Alam ${ }^{4}$, Chad Ellimoottil ${ }^{2,3}$
}

${ }^{1}$ Medical School, ${ }^{2}$ Institute for Healthcare Policy and Innovation, ${ }^{3}$ Department of Urology, ${ }^{4}$ Department of Surgery, ${ }^{5}$ Ross School of Business, University of Michigan, Ann Arbor, MI, USA

Contributions: (I) Conception and design: All authors; (II) Administrative support: All authors; (III) Provision of study materials or patients: D Portney, R Ved, C Ellimoottil; (IV) Collection and assembly of data: All authors; (V) Data analysis and interpretation: DS Portney, C Ellimoottil; (VI) Manuscript writing: All authors; (VII) Final Approval of Manuscript: All authors.

Correspondence to: Chad Ellimoottil, MD, MS. Department of Urology, University of Michigan, 2800 Plymouth Rd. NCRC Bldg. 16, Rm. 151S, Ann Arbor, MI 48109, USA. Email: cellimoo@med.umich.edu.

Background: Expansion of telehealth is a high-priority strategic initiative for many health systems. Surgical clinics' implementation of video visits has been identified as a way to improve patient and provider experience. However, whether using video visits can reduce the cost of an outpatient visit is unknown.

Methods: Prospective case study using time-driven activity-based costing at two outpatient surgical clinics at an academic institution. We conducted stakeholder interviews and in-person observations to map outpatient clinic flow and measure resource utilization of four key steps: check-in, vitals collection and rooming, clinician encounter, and check-out. Finally, we calculated the resource cost for each step using representative salary information to calculate total visit cost.

Results: Video visits did not systematically reduce the amount of time clinicians spent with patients. Mean [standard deviation (SD)] visit costs were as follows: traditional clinic visits, \$26.84 (\$10.13); physician-led video visits, \$27.26 (\$9.69); and physician assistant-led video visits, \$9.86 (\$2.76). There was no significant difference in the total cost associated with physician-led traditional clinic visits and video visits $(\mathrm{P}=0.89)$. However, physician assistant-led video visits were significantly lower cost than physician-led video visits $(\mathrm{P}<0.001)$.

Conclusions: Using physician-led video visits does not reduce the cost of outpatient surgical visits when compared to traditional clinic visits. However, the use of less expensive clinician resources for video visits (e.g., physician-assistants) may yield cost savings for clinics.

Keywords: Telemedicine; telehealth; cost analysis; video visits; provider costs

Received: 10 January 2020; Accepted: 26 May 2020; Published: 05 October 2020.

doi: 10.21037/mhealth-20-33

View this article at: http://dx.doi.org/10.21037/mhealth-20-33

\section{Introduction}

In 2013, just over half of U.S. hospitals used some method of telehealth (1). Since then, telehealth has become increasingly common and varied in its use, including in the clinic setting (2). In $2018,70 \%$ of surveyed healthcare organizations reported telehealth as a top- or high-priority strategic initiative (2), and additional reports show that $83 \%$ of responding healthcare executives were likely to invest in

\footnotetext{
$\wedge$ David S. Portney ORCID: 0000-0003-3931-0463; Vahagn Nikolian ORCID: 0000-0001-6028-8614; Hasan B. Alam ORCID: 0000-00021024-5226.
} 
telehealth in 2017 (3). In 2020, the COVID-19 pandemic caused a paradigm shift in the use of telehealth, significantly increasing its use and reducing regulatory barriers (4).

Video visits, a form of telehealth, allow patients and physicians to communicate via videoconferencing software and have been shown to improve patient experience and access to care (5). Such visits have been used widely in the surgical field, including general surgery and subspecialties, such as urology and otolaryngology (6-13). With the noteworthy enthusiasm for and growing implementation of video visits, a common assumption is that video visits are a lower-cost alternative to traditional clinic visits.

Despite the pace at which health systems are implementing and using video visit technology, its cost-saving value in a clinical setting has not been well-studied from the provider perspective. One presumption is that video visits, which are often conducted between physicians and patients without the use of office staff, can reduce overall clinic costs. However, research on video visits has focused on quality of care and patient costs and experience, rather than clinic costs. It has been shown that post-operative video visits in outpatient surgical clinics do not lead to complications (11). Additionally, multiple studies suggest strong patient willingness to try video visits (14-17), as well as the ability for video visits to reduce appointment and travel times and patients' time away from work $(12,18-20)$. However, these analyses are limited to cost savings to the patient and do not address cost savings for the provider. Robust economic analysis of telemedicine is rare and only a few studies have highlighted the benefits or risks for providers; for instance, increased capacity to schedule appointments through the use of video visits $(8,19-21)$.

Our study aims to fill this gap by understanding the provider perspective on the costs of video visits. To do so, we used a well-established costing methodology called timedriven activity-based costing (TDABC) (22-24).

\section{Methods}

\section{Study population}

Our study assessed the cost of clinic visits and video visits at two outpatient surgery clinics, urology and general surgery, at a large academic medical center. We selected these two clinics because both: (I) have had established video visit programs for over 2 years and are therefore past the basic learning curve; (II) involve multiple faculty members in video visits; and (III) have similar clinic workflows with shared medical assistant and clerical staff. In order to make a more direct comparison with video visits, we included only established patients who were not receiving any procedures while in the office. Henceforth, we group both urology and general surgery in-person clinic visits together as "traditional clinic visits". While both the urology and general surgery clinics use video visits for post-operative patient visits, attending physicians conduct urology video visits, and physician assistants conduct general surgery video visits.

Kaplan and Anderson developed TDABC to accurately calculate the cost of a process (22). After successful implementation in and use by many industries, Kaplan and Porter described TDABC's functionality and use in healthcare as well as seven steps to implement it (23). Since then, many studies have outlined TDABC's use for two primary aims: (I) to inform reimbursement policy in an ecosystem driven by the need to reduce costs and (II) to support operational improvements (24). In our model, we included only variable costs associated with traditional and video visits, examined through the lens of the provider. We excluded fixed costs, such as the investment in telehealth, because these costs had already been made and the goals of our study focused on comparing costs in a stable state. We did not address patient savings (such as driving time or time off work) as these did not directly affect a provider's costs. Additionally, we did not include metrics of quality as our study was strictly focused on costs. Our study used the following steps outlined by Kaplan and Porter with some modifications.

\section{TDABC steps}

\section{Step 1: select the medical condition}

We focused on clinical encounters (established return visits with no procedures) rather than a medical condition. Such deviation from the original definition is common in other studies (24).

\section{Step 2: define the care delivery value chain}

We chose to define our process starting when a patient utilizes clinic resources and ending when a patient stops using them, as our study's aim is for operational improvement rather than informing reimbursement policy (24).

Step 3: develop process maps that include each activity in patient care delivery and incorporate all direct and indirect capacity-supplying resources

We developed process maps using a two-step method: (I) 


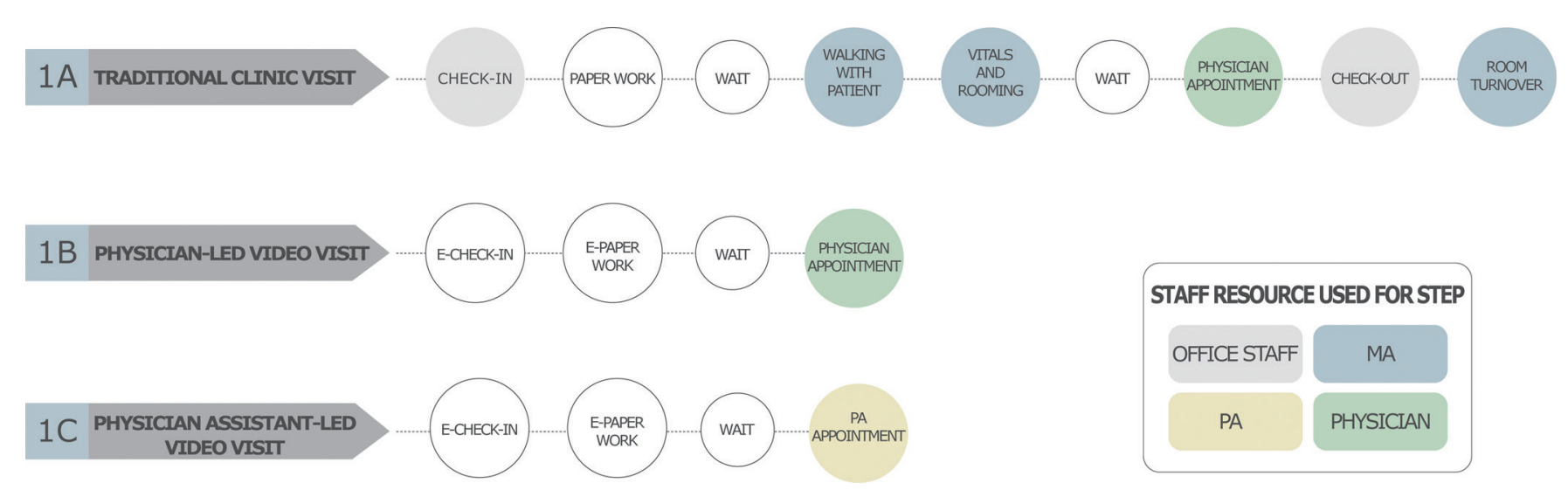

Figure 1 Process maps for the three different types of visits. Process maps for the three types of visits were created through a mix of stakeholder interviews and observational data. Non-resource intensive steps (colored white), such as patient waiting time and e-paperwork, do not incur clinic costs and were excluded from future calculations of cost using time-driven activity-based costing (TDABC). MA, medical assistant; PA, physician assistant.

stakeholder interviews and (II) in-person observations. We performed stakeholder interviews from 2/28/2018 to $9 / 5 / 2018$. The interviews, conducted with physicians, physician assistants, medical assistants, and office managers, sought to understand the patient and staff flow during clinic visits. As a result of these data, we created three process maps (Figure 1) to display the flow of patients and the use of resources through three types of visits: traditional clinic visits, physician-led video visits, and physician assistant-led video visits.

\section{Step 4: obtain time estimates for each process}

We used estimates from stakeholder interviews as the early guides to understanding process time and variation. We identified four key steps as high-impact and highly variable between visits: check-in, vitals collection and rooming, clinician encounter, and check-out. We performed time observations between 4/30/2018 and 11/28/2018. We collected check-in and check-out times by observing the time between the start and end of a patient interaction at the front desk. We collected vitals and rooming times with medical assistants by observing entrance and exit patterns of medical assistants from patient rooms through hallway monitoring. Traditional clinic-visit physician times were collected by observing the start and end times for providers with their patients during various clinic days. Physicianled video visits utilized scribes and provider self-timing for data collection. For physician assistant-led video visits, clinician times for video visits were tracked using a selfreporting Microsoft Excel worksheet. Based on stakeholder interviews, we determined note closing time to be variable between providers, but without significant difference between traditional clinic visits to video visits. As providers tended to separate the activity of note closing from the patient encounter, we did not include note closing in process-flow calculations. Finally, the total patient-in-room times, required for calculation of room resource costs, were obtained from prior work in the same clinics (21).

\section{Step 5: estimate the cost of supplying patient care resources}

We obtained staff salaries and costs, patient room costs, and resource capacities through clinic administrative teams. We averaged medical assistant, front-desk staff, and physician assistant salaries for the departments. Clinic administrators used representative physicians to calculate and allocate average physician salaries. Annual costs for patient rooms were determined and averaged through health system square-footage allocations.

\section{Step 6: estimate the capacity of each resource and calculate the capacity cost rate (CCR)}

We estimated average resource capacities through stakeholder and clinic administrator interviews. Practical capacity was calculated through determining the total time a resource was available for use (theoretical capacity) in conjunction with the percentage of time it was practically used in a valuable way (utilization rate). We calculated theoretical capacities for medical assistants, office staff, and patient rooms through weekly hours. To calculate practical 
Table 1 Resource costs and capacity cost rate calculation

\begin{tabular}{|c|c|c|c|c|c|c|c|}
\hline Resource & $\begin{array}{c}\text { Annual salary }{ }^{\dagger} \\
\text { (\$) }\end{array}$ & $\begin{array}{l}\text { Clinic annual } \\
\text { allocated } \\
\text { salary }{ }^{\ddagger}(\$)\end{array}$ & $\begin{array}{l}\text { Clinic annual } \\
\text { allocated } \\
\text { costs }^{\text {" }}(\$)\end{array}$ & $\begin{array}{c}\text { Theoretical } \\
\text { weekly hours }{ }^{\#}\end{array}$ & $\begin{array}{c}\text { Adjusted } \\
\text { weekly hours }\end{array}$ & $\begin{array}{c}\text { Weeks per } \\
\text { year }\end{array}$ & $\begin{array}{c}\text { Capacity cost } \\
\text { rate }(\$ / \mathrm{min})\end{array}$ \\
\hline Office staff & 37,753 & 37,753 & 49,078 & 40 & 32 & 48.0 & 0.53 \\
\hline Physician & 317,254 & 76,951 & 100,036 & 20.6 & 18.9 & 48.0 & 1.84 \\
\hline Patient room & 3,831 & 3,831 & 3,831 & 50 & 50 & 50.5 & 0.03 \\
\hline
\end{tabular}

${ }^{\dagger}$, salary and cost data came from clinic administrator reports; ${ }^{\ddagger}$, allocation of physicians and physician assistant salaries came from clinic administrator reports and interviews; ${ }^{\S}$, physician assistant cost allocation to video visits was based on a $5 \%$ allocation of monthly salary for fixed e-clinic hours; ", employee resource costs were inflated by a flat fee of $30 \%$ to include benefits; ", weekly clinic hours, utilization percentage, and weeks per year were determined through a combination of interviews and surveys.

capacity, these were subsequently reduced according with a flat $80 \%$ utilization rate in line with the number of breaks allowed per day. Anonymous surveys sent to the physicians allowed for an estimate of utilization rates (92\%), which we then applied to both physicians and physician assistants to yield practical capacities. The calculation of CCR by resource is shown in Table 1 .

\section{Step 7: calculate the total cost of patient care}

We used step times and CCR to calculate the total cost of clinic encounters, where $i$ represents an individual process step.

$$
\text { Total Visit Costs }=\sum\left(\text { time }_{i}\right)\left(C C R_{i}\right)
$$

\section{Outcomes}

The primary outcome of interest is the total cost of an outpatient clinic visit to the provider. We were specifically interested in comparing the difference in average total cost between the three types of visits beings studied: traditional clinic visits, physician-led video visits, and physician assistant-led video visits. All statistical analysis was completed using Stata 15 (StatCorp LLC, College Station, TX, USA).

This study was deemed not regulated by IRB because it was a quality improvement initiative.

\section{Results}

Interview and observational data were combined to create process maps for the three processes of interest, shown in Figure 1. Traditional clinic visits in urology and general surgery clinics had similar clinician times during patient visits (9.96 vs. $10.52 \mathrm{~min}, \mathrm{P}=0.69$ ), and were grouped for further calculations. Table 2 outlines the descriptive data for clinician steps. We found an increased physician time for video visits $[\mu=13.8 \mathrm{~min}$, standard deviation $(\mathrm{SD})=5.3 \mathrm{~min}]$ compared to traditional visits $(\mu=10.2 \mathrm{~min}, \mathrm{SD}=5.3 \mathrm{~min}$ ), though physician assistant-led video visits were associated with slightly less time than both $(\mu=9.7 \mathrm{~min}, \mathrm{SD}=3.0 \mathrm{~min}$ ).

Figure 2 summarizes total visit costs calculated using TDABC. It outlines average clinic costs associated with each type of visit and highlights the non-clinician component of the visit for traditional clinic visits. Approximately $70 \%$ of traditional clinic visit costs is associated with clinicians because physicians are significantly more expensive than other clinic resources, and because physician time is the most time-consuming part of a clinic visit. Approximately $\$ 8.15$ of traditional clinic-visit costs are associated with non-clinician resources which are no longer required during video visits.

Table 3 shows the median, mean, SD, and standard error of costs for each of the three processes. Mean (SD) costs were as follows: traditional clinic visits \$26.84 (\$10.13), physician-led video visits $\$ 27.26$ (\$9.69), and physician assistant-led video visits $\$ 9.86$ (\$2.76). T-tests using unequal variances for difference in means between traditional clinic visits and the physician-led video visits showed no significant difference $(\mathrm{P}=0.89, t=-0.14, \mathrm{df}=21.99)$. Therefore, there are only minor, if any, cost savings associated with physicianled video visits. However, physician assistant-led video visits 
Table 2 Summary of observational data (time in minutes)

\begin{tabular}{lccc}
\hline Statistic & $\begin{array}{c}\text { Traditional clinic visit clinician } \\
\text { time }^{\dagger}\end{array}$ & $\begin{array}{c}\text { Physician-led video visit } \\
\text { clinician time }\end{array}$ & $\begin{array}{c}\text { Physician assistant-led video } \\
\text { visit clinician time }\end{array}$ \\
\hline N & 73 & 14 & 15 \\
Mean & 10.2 & 13.8 & 9.7 \\
St. Dev. & 5.3 & 5.3 & 3.0 \\
Min & 2.0 & 6.0 & 5.0 \\
Max & 33.0 & 24.0 & 13.3 \\
\hline
\end{tabular}

${ }^{\dagger}$, traditional clinic visit times are only available as whole minutes due to method of collection.

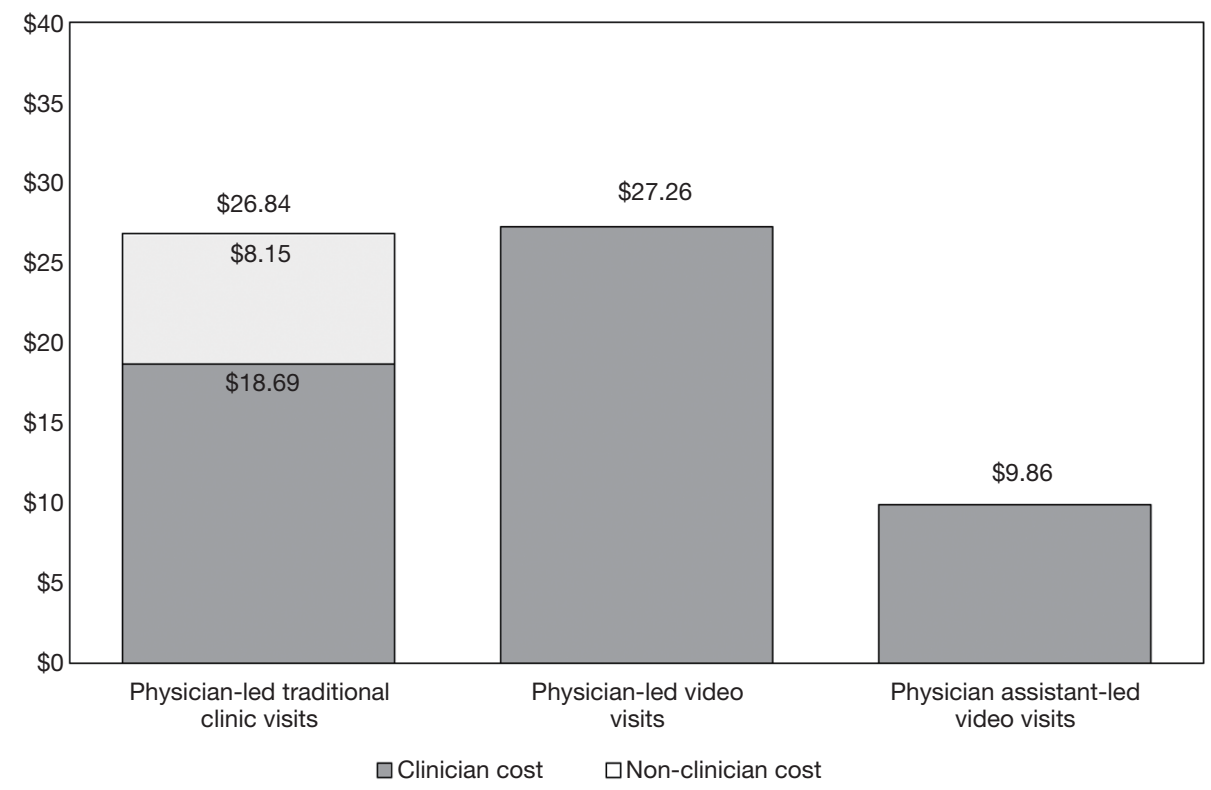

Figure 2 Visit costs by visit type and resource type. Visit costs compared between traditional clinic visits and physician-led video visits show minimal difference. However, physician assistant-led video visits were on average $\$ 17.40$ cheaper than physician-led video visits. Data was obtained through in-person observation of each visit and calculated using time-driven activity-based costing.

were significantly $(\mathrm{P}<0.001)$ less costly than physician-led video visits $(t=6.48, \mathrm{df}=14.96)$, illustrating a reduction in total cost of physician assistant-led video visits compared to physician-led video visits. Examination of the variation within individual processes yields a wide SD in costs for physician-led visits showing high variability between individual providers and visits. On the other hand, the SD for physician assistant-led video visits is much smaller, even as a percentage of total visit costs.

\section{Discussion}

Our study highlights the overwhelming role that physician costs, which comprise $70 \%$ of total visit costs, play in a clinic visit. Moreover, we show that a video visit may be a cost-effective alternative to clinic visits, contingent upon the structure of the visits. Physician-led video visits were approximately the same cost as traditional clinic visits, but physician assistant-led video visits reduced visit costs by greater than $60 \%$ compared to physician-led video visits. Collectively, these findings suggest that video visits led by physicians do not inherently lead to cost savings; however, they can be a cost-effective alternative when utilizing lower cost resources.

Our finding that physician costs are the predominant driver of costs in a clinic setting is consistent with a prior 
Table 3 Time-driven activity-based costs (TDABC) of each process

\begin{tabular}{lccc}
\hline Process & Traditional clinic visits (\$) & Physician-led video visits (\$) & $\begin{array}{c}\text { Physician assistant-led video } \\
\text { visits (\$) }\end{array}$ \\
\hline Median cost $^{\dagger}$ & 25.46 & 24.86 & 9.50 \\
Mean cost $^{\ddagger}$ & 26.84 & 27.26 & 9.86 \\
St. Dev. of costs & 10.13 & 9.69 & 2.76 \\
St. error of costs & 1.20 & 2.59 & 0.71 \\
\hline
\end{tabular}

${ }^{\dagger}$, median costs were calculated by a sum product of the median time for each step multiplied by the capacity cost rate for the given resource; ${ }^{\ddagger}$, mean costs were calculated similarly to median costs, but utilizing the means rather than the median times for each step; ${ }^{\S}$, capacity cost rates were multiplied by individual step standard deviations to calculate a standard deviation of the step costs. Standard deviations were then converted to variance and combined for each step of the process of interest in order to calculate the cost standard deviation for the entire process; ", standard error of costs was sought to understand the estimated difference between average costs. This calculation was similar to the standard deviation of costs calculation, but started with process time standard errors rather than standard deviation.

study by Yun et al. This study, which utilized TDABC, showed that physicians account for $68 \%$ of staff costs for a chest pain presentation in the emergency department (25). Our study adds to the current literature by focusing on outpatient surgical clinics, where we show that traditional clinic visits are similarly driven by clinician costs.

Our findings surrounding the limited cost savings of video visits is generally consistent with prior literature. We found no significant difference between the costs of traditional clinic visits and physician-led video visits $(\mathrm{P}=0.89)$. Theodore et al. examined the transaction costs of pain consults and found that the per patient costs for video visits and traditional visits were more or less the same (26). While studies like that of Zholudev et al. noted savings using video visits, only a minority percentage of these savings were associated with provider costs as it was a VA-led comparison with shared savings from patient transportation making up the majority (27). On the other hand, other studies showed video consults had a reduced per-visit cost as well as the ability to accumulate significant health system savings over a 5 -year period $(28,29)$. In our study, while the physicianled video visits were approximately equal to traditional visits in terms of per-visit costs, physician assistant-led video visits were able to reduce the per-visit costs by $64 \%$ compared to physician-led video visits. Our study furthers the current literature base by illustrating that the profitability of video visits for providers is reliant on the delivery structure, as they do not create dramatic time savings for clinicians.

Our study has several limitations. First, it took place at a single institution and examined video visits in the only two surgical clinics with well-established video visit programs. As such, our findings may not be generalizable to other specialties. However, our findings identified drivers of costefficiency for video visits, and these will likely hold true for most surgical practices. Secondly, we were unable to compare physician assistant traditional clinic visits directly with video visits or physician traditional clinic visits because the study's participating clinics did not utilize physician assistants in traditional clinic visits. While we did not make this comparison, it is assumed that shifting traditional clinic visits from physicians to physician assistants would yield a similar level of cost savings as was the case for physician video visits to physician assistant-led video visits. Thirdly, given the limited number of video visits utilized at our institution, our study had a small sample size. In result, our study concluded an insignificant cost difference between traditional clinic visits and physician-led video visits. Further work utilizing a large number of visits may elucidate subtle differences. Finally, our cost estimates did not take into account the capital costs associated with setting up a video visit program, which would vary greatly between surgical practices based on size and existing telehealth infrastructure. With the costs evaluated being standard practice and insurance independent, however, this variability is greatly minimized allowing for the gathered data to be applicable to most developed health systems.

These limitations notwithstanding, our findings can help providers make evidence-based decisions surrounding the implementation of video visits. Our study suggests that the anticipated cost savings from video visits are generally insignificant when physicians are conducting them, but substantial when lower-cost providers substitute into provider roles. Therefore, though it has been believed that video visits reduce costs, the reality is more ambiguous and 
variable. And while $83 \%$ of surveyed respondents described cost savings as a priority for telehealth program objectives, it was still only the eighth-highest priority out of 16 objectives, leading observers to note the relative importance of other factors such as improving patient outcomes, access, and satisfaction (2). Therefore, reduced cost savings associated with video visits may not preclude increased video visit usage in the future.

Our study findings can be added to a growing base of literature surrounding the costs and benefits of telehealth and video visits. Our research adds to the narrow body of literature examining the benefits and risks of telehealth for providers by examining the cost savings of video visit implementation. Further work can build on this theme by measuring cost savings in a larger range of delivery models as well as examining the opportunity for increased provider revenue through the use of video visit technology. Additional studies can consider the impact of technology and infrastructure investment costs required for the implementation of video visits. In the end, the implications of this work will guide provider, payer, and policymaker decisions surrounding the use of new technology in healthcare and ultimately impact the way patients receive care in the future.

\section{Acknowledgments}

Funding: This research was supported by the Blue Cross Blue Shield of Michigan Foundation (002713.SAP to Mr. Portney) and by the Telehealth Research Incubator project grant (MPrOVE Research Challenge Grant to Dr. Ellimoottil). No funding organization was involved in the design and conduct of the study; collection, management, analysis, and interpretation of the data; preparation, review, or approval of the manuscript; and decision to submit the manuscript for publication.

\section{Footnote}

Data Sharing Statement: Available at http://dx.doi. org/10.21037/mhealth-20-33

Peer Review File: Available at http://dx.doi.org/10.21037/ mhealth-20-33

Conflicts of Interest: All authors have completed the ICMJE uniform disclosure form (available at http://dx.doi. org/10.21037/mhealth-20-33). DSP reports grants from
Blue Cross Blue Shield of Michigan Foundation, during the conduct of the study; CE reports grants from MPrOVE Research Challenge Grant, during the conduct of the study. The other authors have no conflicts of interest to declare.

Ethical Statement: The authors are accountable for all aspects of the work in ensuring that questions related to the accuracy or integrity of any part of the work are appropriately investigated and resolved.

Open Access Statement: This is an Open Access article distributed in accordance with the Creative Commons Attribution-NonCommercial-NoDerivs 4.0 International License (CC BY-NC-ND 4.0), which permits the noncommercial replication and distribution of the article with the strict proviso that no changes or edits are made and the original work is properly cited (including links to both the formal publication through the relevant DOI and the license). See: https://creativecommons.org/licenses/by-nc-nd/4.0/.

\section{References}

1. American Hospital Association. The Promise of Telehealth for Hospitals, Health Systems and Their Communities. American Hospital Association. 2015. Available online: https://www.aha.org/guidesreports/2015-01-20promise-telehealth-hospitals-health-systems-and-theircommunities

2. REACH Health. 2018 U.S. Telemedicine Industry Benchmark Survey. 2018;(March). Available online: http://reachhealth.com/wp-content/uploads/2018_US_ Telemedicine_Industry_Benchmark_Survey_REACH_ Health2018031520.pdf

3. Goerlich C. 2018 Telehealth Industry Trends. 2018. Available online: https://www.advisory. com/research/population-health-advisor/events/ webconferences/2018/2018-telehealth-industry-trends/ ondemand

4. Webster P. Virtual health care in the era of COVID-19. Lancet 2020;395:1180-1.

5. Modi PK, Portney D, Hollenbeck BK, et al. Engaging telehealth to drive value-based urology. Curr Opin Urol 2018;28:342-7.

6. Yoder LH, McFall DC, Cancio LC. Use of the videophone to collect quality of life data from burn patients. Int J Burns Trauma 2012;2:135-44.

7. Eisenberg D, Hwa K, Wren SM. Telephone followup by a midlevel provider after laparoscopic inguinal 
hernia repair instead of face-to-face clinic visit. JSLS 2015;19:e2014.00205.

8. Hwa K, Wren SM. Telehealth follow-up in lieu of postoperative clinic visit for ambulatory surgery: Results of a pilot program. JAMA Surg 2013;148:823-7.

9. Sathiyakumar V, Apfeld JC, Obremskey WT, et al. Prospective randomized controlled trial using telemedicine for follow-ups in an orthopedic trauma population: A pilot study. J Orthop Trauma 2015;29:e139-45.

10. Urquhart AC, Antoniotti NM, Berg RL. TelemedicineAn efficient and cost-effective approach in parathyroid surgery. Laryngoscope 2011;121:1422-5.

11. Gunter RL, Chouinard S, Fernandes-Taylor S, et al. Current Use of Telemedicine for Post-Discharge Surgical Care: A Systematic Review. J Am Coll Surg 2016;222:915-27.

12. Viers BR, Lightner DJ, Rivera ME, et al. Efficiency, Satisfaction, and Costs for Remote Video Visits Following Radical Prostatectomy: A Randomized Controlled Trial. Eur Urol 2015;68:729-35.

13. Hakim AA, Kellish AS, Atabek U, et al. Implications for the use of telehealth in surgical patients during the COVID-19 pandemic. Am J Surg 2020;220:48-9.

14. Andino JJ, Guduguntla V, Weizer A, et al. Examining the Value of Video Visits to Patients in an Outpatient Urology Clinic. Urology 2017;110:31-5.

15. Powell RE, Henstenburg JM, Cooper G, et al. Patient perceptions of telehealth primary care video visits. Ann Fam Med 2017;15:225-9.

16. Viers BR, Pruthi S, Rivera ME, et al. Ambulatory, Officebased, and Geriatric Urology Are Patients Willing to Engage in Telemedicine for Their Care: A Survey of Preuse Perceptions and Acceptance of Remote Video Visits in a Urological Patient Population. Urology 2015;85:1233-9.

17. American Well Corporation. Telehealth Index: 2017 Consumer Survey. 2017. Available online: http:// go.americanwell.com/rs/335-QLG-882/images/ American_Well_Telehealth_Index_2017_Consumer_

doi: $10.21037 /$ mhealth-20-33

Cite this article as: Portney DS, Ved R, Nikolian V, Wei A, Buchmueller T, Killaly B, Alam HB, Ellimoottil C. Understanding the cost savings of video visits in outpatient surgical clinics. mHealth 2020;6:32.

\section{Survey.pdf}

18. Ellimoottil C, Boxer RJ. Bringing Surgical Care to the Home Through Video Visits. JAMA Surg 2018;153:177.

19. Chu S, Boxer R, Madison P, et al. Veterans Affairs Telemedicine: Bringing Urologic Care to Remote Clinics. Urology 2015;86:255-60.

20. Russo JE, McCool RR, Davies L. VA Telemedicine: An Analysis of Cost and Time Savings. Telemed J E Health 2016;22:209-15.

21. Nikolian VC, Williams AM, Jacobs BN, et al. Pilot Study to Evaluate the Safety, Feasibility, and Financial Implications of a Postoperative Telemedicine Program. Ann Surg 2018;268:700-7.

22. Kaplan RS, Anderson SR. Time-driven activity-based costing. Harv Bus Rev 2004;82:131-8, 150.

23. Kaplan RS, Porter ME. How to solve the cost crisis in health care. Harv Bus Rev 2011;89:46-52, 54, 56-61 passim.

24. Keel G, Savage C, Rafiq M, et al. Time-driven activitybased costing in health care: A systematic review of the literature. Health Policy 2017;121:755-63.

25. Yun BJ, Prabhakar AM, Warsh J, et al. Time-Driven Activity-Based Costing in Emergency Medicine. Ann Emerg Med 2016;67:765-72.

26. Theodore BR, Whittington J, Towle C, et al. Transaction Cost Analysis of In-Clinic Versus Telehealth Consultations for Chronic Pain: Preliminary Evidence for Rapid and Affordable Access to Interdisciplinary Collaborative Consultation. Pain Med 2015;16:1045-56.

27. Zholudev V, Safir IJ, Painter MN, et al. Comparative Cost Analysis: Teleurology vs Conventional Face-to-Face Clinics. Urology 2018;113:40-4.

28. Xu CQ, Smith AC, Scuffham PA, et al. A cost minimisation analysis of a telepaediatric otolaryngology service. BMC Health Serv Res 2008;8:30.

29. Smith AC, Scuffham P, Wootton R. The costs and potential savings of a novel telepaediatric service in Queensland. BMC Health Serv Res 2007;7:35. 